\title{
Statistics for 3-letter patterns with repetitions in compositions
}

\author{
Armend Sh. Shabani Rexhep Gjergji
}

Department of Mathematics, University of Prishtina

received $5^{\text {th }}$ May 2015, revised $21^{\text {st }}$ June 2015, $7^{\text {th }}$ Apr. 2016, accepted $11^{\text {th }}$ Apr. 2016.

A composition $\pi=\pi_{1} \pi_{2} \cdots \pi_{m}$ of a positive integer $n$ is an ordered collection of one or more positive integers whose sum is $n$. The number of summands, namely $m$, is called the number of parts of $\pi$. Using linear algebra, we determine formulas for generating functions that count compositions of $n$ with $m$ parts, according to the number of occurrences of the subword pattern $\tau$, and according to the sum, over all occurrences of $\tau$, of the first integers in their respective occurrences, where $\tau$ is any pattern of length three with exactly 2 distinct letters.

Keywords: Subwords, generating functions, Cramer's method.

\section{Introduction}

A composition $\pi=\pi_{1} \pi_{2} \cdots \pi_{m}$ of a positive integer $n \in \mathbb{N}$ is an ordered collection of $m$ positive integers whose sum is $n$. The number $m$ of summands is called the number of parts of $\pi$ and is denoted by $\operatorname{ord}(\pi)$. For example, the compositions of 3 are 3,12, 21 and 111. We denote the set of compositions of $n$ by $\mathcal{C}_{n}$, and the set of compositions of $n$ with parts in $[d]=\{1,2, \ldots, d\}$ by $\mathcal{C}_{n ; d}$. Clearly, the number of compositions of $n$ is given by $\left|\mathcal{C}_{n}\right|=2^{n-1}$ (for instance, see [6]).

Let $\pi=\pi_{1} \pi_{2} \cdots \pi_{m}$ be any composition of $n$ with exactly $m$ parts in $[d]$ and let $\sigma=\sigma_{1} \sigma_{2} \cdots \sigma_{s}$ be any word of length $s$, where $m \geq s$. An occurrence of $\sigma$ in $\pi$ is a subword $\pi_{i} \pi_{i+1} \cdots \pi_{i+s-1}$ which is order isomorphic to $\sigma$, i.e., $\pi_{i-1+a}<\pi_{i-1+b}$ if and only if $\sigma_{a}<\sigma_{b}$ for all $1 \leq a<b \leq s$. Here, the word $\sigma$ is usually called a pattern of length $s$ (or an $s$-letter pattern). We denote the number of the occurrences of $\sigma$ in $\pi$ by $\operatorname{occ}_{\sigma}(\pi)$ and we denote the sum of the values of the first letters over all occurrences of $\sigma$ in $\pi$ by $s f l_{\sigma}(\pi)$. For example, the occurrences of the pattern 121 in the composition $\pi=12122421$ of 15 are 121 and 242 , so occ $121(\pi)=2$ and $\operatorname{sfl}_{121}(\pi)=1+2=3$.

In last decade, statistics on compositions (a statistic on set $A$ is a function $A \rightarrow \mathbb{N}_{0}=\{0,1,2, \ldots\}$ ) has been received a lot of attention (see [6] and references therein). For instance, Heubach and Mansour [5] studied the generating function for the number of compositions of $n$ with exactly $m$ parts according to the number of occurrences of the patterns 11, 12 and 21. Mansour and Sirhan [8] discussed the generating function for the number of compositions of $n$ with exactly $m$ parts according to the number of occurrences of several families of patterns (for other reference, see also [1, 3, 4] and references therein). 
Recently, by using linear algebra, Askaly and Mansour [2] determined formulas for generating functions that count compositions of $n$ with $m$ parts, according to the numbers of pattern $\tau$ of length 2 , and according to the sum, over all occurrences of $\tau$, of the first integers in their respective occurrences.

Let $F_{\tau}=F_{\tau}(x, y, u, v)$ be the generating function for the number of compositions of $n$ with parts in $[d]$ according to the statistics ord, $\mathrm{occ}_{\tau}$ and $\mathrm{sfl}_{\tau}$, that is

$$
F_{\tau ; d}=\sum_{n \geq 0} \sum_{\pi \in \mathcal{C}_{n ; d}} x^{n} y^{\mathrm{ord}(\pi)} u^{\mathrm{sfl}_{\tau}(\pi)} v^{\mathrm{occ}_{\tau}(\pi)}
$$

In this paper, by using linear algebra, we extend the results of [2] to derive the generating functions for the number of compositions, the number of parts, and the statistics occ $\tau$ and $\mathrm{sfl}_{\tau}$. This unified framework generalizes earlier works.

The paper is organized as follows. In the next section, we obtain generating functions for the number of compositions of $n$ with parts in $[d]$, according to the above mentioned statistics. Finally, in the last section, we use our results to derive the average sum of the values of the first letters of the occurrences of $\sigma$ in words of $[d]^{n}$, where $\sigma \in\{112,121,122,211,212,221\}$.

In order to achieve our results, we need the following notation. We define

$$
F_{\tau}\left(a_{1} a_{2} \cdots a_{s}\right)=F_{\tau}\left(x, y, u, v \mid a_{1} a_{2} \cdots a_{s}\right)
$$

to be the generating function for the number of compositions $\pi=a_{1} a_{2} \cdots a_{s} \pi^{\prime}$ of $n$ with parts in $[d]$ according to the statistics ord, $\mathrm{occ}_{\tau}$ and $\mathrm{sfl}_{\tau}$, that is

$$
F_{\tau ; d}\left(a_{1} a_{2} \cdots a_{s}\right)=\sum_{n \geq 0} \sum_{\pi=a_{1} a_{2} \cdots a_{s} \pi^{\prime} \in \mathcal{C}_{n ; d}} x^{n} y^{\mathrm{ord}(\pi)} u^{\mathrm{sfl}_{\tau}(\pi)} v^{\mathrm{occ}_{\tau}(\pi)} .
$$

Clearly,

$$
F_{\tau ; d}=1+\sum_{j=1}^{d} F_{\tau ; d}(j) .
$$

We will conclude the paper with a short description on future work.

\section{Three letters pattern}

This section is divided into several subsections, where in each subsection we study a fixed pattern from the set $\{112,121,122,211,212,221\}$.

\subsection{The case $\tau=112$}

In this subsection, we find an explicit formula for the generating function $F_{112 ; d}$. Firstly, we find a recurrence relation for the generating function $F_{112 ; d}(a)$. By the definitions, we have

$$
\begin{aligned}
F_{112 ; d}(a) & =x^{a} y+\sum_{b=1, b \neq a}^{d} F_{112 ; d}(a b)+F_{112 ; d}(a a) \\
& =x^{a} y+x^{a} y \sum_{b=1, b \neq a}^{d} F_{112 ; d}(b)+F_{112 ; d}(a a),
\end{aligned}
$$


which, by (1), implies

$$
F_{112 ; d}(a)=x^{a} y\left(F_{112 ; d}-F_{112 ; d}(a)\right)+F_{112 ; d}(a a) .
$$

Now, we express $F_{112 ; d}(a a)$ in terms of generating functions $F_{112 ; d}(j)$. Again, by definitions, we have

$$
\begin{aligned}
F_{112 ; d}(a a) & =x^{2 a} y^{2}+\sum_{j=1}^{a-1} F_{112 ; d}(a a j)+F_{112 ; d}(a a a)+\sum_{j=a+1}^{d} F_{112 ; d}(a a j) \\
& =x^{2 a} y^{2}+x^{2 a} y^{2} \sum_{j=1}^{a-1} F_{112 ; d}(j)+x^{a} y F_{112 ; d}(a a)+x^{2 a} y^{2} u^{a} v \sum_{j=a+1}^{d} F_{112 ; d}(j),
\end{aligned}
$$

which gives

$$
F_{112 ; d}(a a)=\frac{x^{2 a} y^{2}}{1-x^{a} y}\left(1+\sum_{j=1}^{a-1} F_{112 ; d}(j)+u^{a} v \sum_{j=a+1}^{d} F_{112 ; d}(j)\right)
$$

Therefore, by [1], we obtain

$$
F_{112 ; d}(a a)=\frac{x^{2 a} y^{2}}{1-x^{a} y}\left(F_{112 ; d}-F_{112 ; d}(a)+\left(u^{a} v-1\right) \sum_{j=a+1}^{d} F_{112 ; d}(j)\right) .
$$

By substituting (3) into (2), we get

$$
F_{112 ; d}(a)=x^{a} y\left(F_{112 ; d}-F_{112 ; d}(a)\right)+\frac{x^{2 a} y^{2}}{1-x^{a} y}\left(F_{112 ; d}-F_{112 ; d}(a)+\left(u^{a} v-1\right) \sum_{j=a+1}^{d} F_{112 ; d}(j)\right) \text {, }
$$

which is equivalent to

$$
F_{112 ; d}(a)=x^{a} y F_{112 ; d}-x^{2 a} y^{2}\left(1-u^{a} v\right) \sum_{j=a+1}^{d} F_{112 ; d}(j) .
$$

Let $\alpha_{a}=x^{2 a} y^{2}\left(1-u^{a} v\right)$ and $\beta_{a}=x^{a} y F_{112 ; d}$. Then (4) can be written as

$$
\mathbf{M}_{112 ; d} x_{112 ; d}=v_{112 ; d}
$$

where $v_{112 ; d}=\left(\beta_{1}, \ldots, \beta_{d}\right)^{T}, x_{112 ; d}=\left(F_{112 ; d}(1), \ldots, F_{112 ; d}(d)\right)^{T}$, and $\mathbf{M}_{112 ; d}=\left(m_{i j}\right)_{1 \leq i, j \leq d}$ with

$$
m_{i j}= \begin{cases}\alpha_{i}, & i<j \\ 1, & i=j \\ 0, & i>j\end{cases}
$$

We solve this system by Cramer's method and obtain

$$
F_{112 ; d}(a)=\frac{\operatorname{det} \mathbf{M}_{112 ; d}^{(a)}}{\operatorname{det} \mathbf{M}_{112 ; d}}=\operatorname{det} \mathbf{M}_{112 ; d}^{(a)},
$$


where $\mathbf{M}_{112 ; d}^{(a)}$ is a matrix obtained from the matrix $\mathbf{M}_{112 ; d}$ by replacing the $a$-th column by the vector $v_{112 ; d}$. By the definitions, we have (we omit the zeros)

$$
\begin{aligned}
F_{112 ; d}(a) & =\operatorname{det}\left(\begin{array}{lllll}
\beta_{a} & \alpha_{a} & \alpha_{a} & \cdots & \alpha_{a} \\
\beta_{a+1} & 1 & \alpha_{a+1} & \cdots & \alpha_{a+1} \\
\beta_{a+2} & & 1 & \cdots & \alpha_{a+2} \\
\vdots & & \ddots & \\
\beta_{d} & & & 1
\end{array}\right) \\
& =\sum_{j=0}^{d-a}(-1)^{j} \beta_{a+j} \operatorname{det}\left(\begin{array}{lllll}
\alpha_{a} & \alpha_{a} & \alpha_{a} & \cdots & \alpha_{a} \\
1 & \alpha_{a+1} & \alpha_{a+1} & \cdots & \alpha_{a+1} \\
& 1 & \alpha_{a+2} & \cdots & \alpha_{a+2} \\
& & \ddots & \ddots & \vdots \\
& & & 1 & \alpha_{a+j-1}
\end{array}\right) .
\end{aligned}
$$

By simple induction on $j \geq 1$, one can show

$$
\operatorname{det}\left(\begin{array}{lllll}
\alpha_{a} & \alpha_{a} & \alpha_{a} & \cdots & \alpha_{a} \\
1 & \alpha_{a+1} & \alpha_{a+1} & \cdots & \alpha_{a+1} \\
& 1 & \alpha_{a+2} & \cdots & \alpha_{a+2} \\
& & \ddots & \ddots & \vdots \\
& & & 1 & \alpha_{a+j-1}
\end{array}\right)=\alpha_{a} \prod_{i=1}^{j-1}\left(\alpha_{a+i}-1\right) .
$$

Hence, we have

$$
F_{112 ; d}(a)=\beta_{a}+\sum_{j=1}^{d-a}(-1)^{j} \beta_{a+j} \alpha_{a} \prod_{i=1}^{j-1}\left(\alpha_{a+i}-1\right) .
$$

By substituting this into (1), we obtain

$$
F_{112 ; d}=1+\sum_{a=1}^{d}\left(\beta_{a}+\sum_{j=1}^{d-a}(-1)^{j} \beta_{a+j} \alpha_{a} \prod_{i=1}^{j-1}\left(\alpha_{a+i}-1\right)\right) .
$$

On the other hand,

$$
\begin{aligned}
\sum_{a=1}^{d}\left(\beta_{a}+\sum_{j=1}^{d-a}(-1)^{j} \beta_{a+j} \alpha_{a} \prod_{i=1}^{j-1}\left(\alpha_{a+i}-1\right)\right) & =\beta_{1}+\sum_{k=2}^{d} \beta_{k}\left(1+\sum_{j=1}^{k-1}(-1)^{j} \alpha_{k-j} \prod_{i=1}^{j-1}\left(\alpha_{k-j+i}-1\right)\right) \\
& =\beta_{1}+\sum_{k=2}^{d}(-1)^{k-1} \beta_{k} \prod_{i=1}^{k-1}\left(\alpha_{i}-1\right) \\
& =\sum_{k=1}^{d}(-1)^{k-1} \beta_{k} \prod_{i=1}^{k-1}\left(\alpha_{i}-1\right) .
\end{aligned}
$$


Hence, by definitions of $\alpha_{a}$ and $\beta_{a}$, we have

$$
\begin{aligned}
F_{112 ; d} & =1+\sum_{k=1}^{d}(-1)^{k-1} \beta_{k} \prod_{i=1}^{k-1}\left(\alpha_{i}-1\right) \\
& =1+F_{112 ; d} \sum_{k=1}^{d}(-1)^{k-1} x^{k} y \prod_{i=1}^{k-1}\left(x^{2 i} y^{2}\left(1-u^{i} v\right)-1\right) \\
& =1+F_{112 ; d} \sum_{k=1}^{d} x^{k} y \prod_{i=1}^{k-1}\left(1-x^{2 i} y^{2}\left(1-u^{i} v\right)\right)
\end{aligned}
$$

which gives the following result.

Theorem 2.1 The generating function $F_{112 ; d}(x, y, u, v)$ is given by

$$
F_{112 ; d}(x, y, u, v)=\frac{1}{1-\sum_{k=1}^{d} x^{k} y \prod_{i=1}^{k-1}\left(1-x^{2 i} y^{2}\left(1-u^{i} v\right)\right)}
$$

By taking $d \rightarrow \infty$ in Theorem 2.1, we obtain the main result of this section.

Theorem 2.2 The generating function $F_{112}(x, y, u, v)$ is given by

$$
F_{112}(x, y, u, v)=\frac{1}{1-\sum_{k \geq 1} x^{k} y \prod_{i=1}^{k-1}\left(1-x^{2 i} y^{2}\left(1-u^{i} v\right)\right)}
$$

\subsection{The case $\tau=122$}

In this subsection, we find an explicit formula for the generating function $F_{122 ; d}$. By using similar arguments as in the previous section, we have

$$
F_{122 ; d}(a)=x^{a} y+x^{a} y \sum_{j=1}^{a} F_{122 ; d}(j)+\sum_{j=a+1}^{d} F_{122 ; d}(a j)
$$

Now, we express $F_{122 ; d}(a k)$ with $a<k \leq d$ in terms of generating functions $F_{122 ; d}(j)$. Again, we have

$$
\begin{aligned}
F_{122 ; d}(a k) & =x^{a+k} y^{2}+x^{a+k} y^{2} \sum_{j=1}^{k-1} F_{122 ; d}(j)+x^{a+k} y^{2} u^{a} v F_{122 ; d}(k)+x^{a} y \sum_{j=k+1}^{d} F_{122 ; d}(k j) \\
& =x^{a} y\left(x^{k} y+x^{k} y \sum_{j=1}^{k} F_{122 ; d}(j)+x^{k} y\left(u^{a} v-1\right) F_{122 ; d}(k)+\sum_{j=k+1}^{d} F_{122 ; d}(k j)\right)
\end{aligned}
$$

which, by (5), gives

$$
F_{122 ; d}(a k)=x^{a} y\left(1+x^{k} y\left(u^{a} v-1\right)\right) F_{122 ; d}(k)
$$


Therefore, by substituting this into (5), we obtain

$$
F_{122 ; d}(a)=x^{a} y F_{122 ; d}-x^{a} y^{2}\left(1-u^{a} v\right) \sum_{j=a+1}^{d} x^{j} F_{122 ; d}(j) .
$$

Let $\alpha_{a}=x^{2 a+1} y^{2}\left(1-u^{a} v\right)$ and $\beta_{a}=x^{a} y F_{122 ; d}$. Then $(6)$ can be written as

$$
\mathbf{M}_{122 ; d} x_{122 ; d}=v_{122 ; d}
$$

where $v_{122 ; d}=\left(\beta_{1}, \ldots, \beta_{d}\right)^{T}, x_{122 ; d}=\left(F_{122 ; d}(1), \ldots, F_{122 ; d}(d)\right)^{T}$, and $\mathbf{M}_{122 ; d}=\left(m_{i j}\right)_{1 \leq i, j \leq d}$ with

$$
m_{i j}= \begin{cases}x^{j-i-1} \alpha_{i}, & i<j \\ 1, & i=j . \\ 0, & i>j\end{cases}
$$

We solve this system by Cramer's method and obtain

$$
F_{122 ; d}(a)=\frac{\operatorname{det} \mathbf{M}_{122 ; d}^{(a)}}{\operatorname{det} \mathbf{M}_{122 ; d}}=\operatorname{det}_{122 ; d}^{(a)},
$$

where $\mathbf{M}_{122 ; d}^{(a)}$ is a matrix obtained from the matrix $\mathbf{M}_{122 ; d}$ by replacing the $a$-th column by the vector $v_{122 ; d}$. By the definitions, we have (we omit the zeros)

$$
\begin{aligned}
F_{122 ; d}(a) & =\operatorname{det}\left(\begin{array}{lllll}
\beta_{a} & \alpha_{a} & x \alpha_{a} & \cdots & x^{d-a-1} \alpha_{a} \\
\beta_{a+1} & 1 & \alpha_{a+1} & \cdots & x^{d-a-2} \alpha_{a+1} \\
\beta_{a+2} & & 1 & \cdots & x^{d-a-3} \alpha_{a+2} \\
\vdots & & \ddots & & \\
\beta_{d} & & & 1 &
\end{array}\right) \\
& =\sum_{j=0}^{d-a}(-1)^{j} \beta_{a+j} \operatorname{det}\left(\begin{array}{llllll}
\alpha_{a} & x \alpha_{a} & x^{2} \alpha_{a} & \cdots & x^{j-1} \alpha_{a} \\
1 & \alpha_{a+1} & x \alpha_{a+1} & \cdots & x^{j-2} \alpha_{a+1} \\
& 1 & \alpha_{a+2} & \cdots & x^{j-3} \alpha_{a+2} \\
& & \ddots & \ddots & \vdots \\
& & & & 1 & \alpha_{a+j-1}
\end{array}\right) .
\end{aligned}
$$

By simple induction on $j \geq 1$, one can show

$$
\operatorname{det}\left(\begin{array}{lllll}
\alpha_{a} & x \alpha_{a} & x^{2} \alpha_{a} & \cdots & x^{j-1} \alpha_{a} \\
1 & \alpha_{a+1} & x \alpha_{a+1} & \cdots & x^{j-2} \alpha_{a+1} \\
& 1 & \alpha_{a+2} & \cdots & x^{j-3} \alpha_{a+2} \\
& & \ddots & \ddots & \vdots \\
& & & 1 & \alpha_{a+j-1}
\end{array}\right)=\alpha_{a} \prod_{i=1}^{j-1}\left(\alpha_{a+i}-x\right) .
$$

Hence, we have

$$
F_{122 ; d}(a)=\beta_{a}+\sum_{j=1}^{d-a}(-1)^{j} \beta_{a+j} \alpha_{a} \prod_{i=1}^{j-1}\left(\alpha_{a+i}-x\right) .
$$


By substituting this into (1), we obtain

$$
F_{122 ; d}=1+\sum_{a=1}^{d}\left(\beta_{a}+\sum_{j=1}^{d-a}(-1)^{j} \beta_{a+j} \alpha_{a} \prod_{i=1}^{j-1}\left(\alpha_{a+i}-x\right)\right)
$$

Hence, by definitions of $\alpha_{a}$ and $\beta_{a}$, we have

$$
F_{122 ; d}=1+F_{122 ; d} \sum_{a=1}^{d}\left(x^{a} y-\sum_{j=a+1}^{d} x^{a+2 j} y^{3}\left(1-u^{a} v\right) \prod_{i=a+1}^{j-1}\left(1-x^{2 i} y^{2}\left(1-u^{i} v\right)\right)\right) .
$$

which gives the following result.

Theorem 2.3 The generating function $F_{122 ; d}(x, y, u, v)$ is given by

$F_{122 ; d}(x, y, u, v)=\frac{1}{1-\sum_{a=1}^{d}\left(x^{a} y-\sum_{j=a+1}^{d} x^{a+2 j} y^{3}\left(1-u^{a} v\right) \prod_{i=a+1}^{j-1}\left(1-x^{2 i} y^{2}\left(1-u^{i} v\right)\right)\right)}$.

By taking $d \rightarrow \infty$ in Theorem 2.1, we obtain the main result of this section.

Theorem 2.4 The generating function $F_{122}(x, y, u, v)$ is given by

$F_{122}(x, y, u, v)=\frac{1}{1-\sum_{a \geq 1}\left(x^{a} y-\sum_{j \geq a+1} x^{a+2 j} y^{3}\left(1-u^{a} v\right) \prod_{i=a+1}^{j-1}\left(1-x^{2 i} y^{2}\left(1-u^{i} v\right)\right)\right)}$.

\subsection{The case $\tau=221$}

In this subsection, we find an explicit formula for the generating function $F_{221 ; d}$. By using similar techniques as showed in the previous subsection, we obtain

$$
F_{221 ; d}(a)=x^{a} y\left(F_{221 ; d}-F_{221 ; d}(a)\right)+F_{221 ; d}(a a)
$$

and

$$
F_{221 ; d}(a a)=\frac{x^{2 a} y^{2}}{1-x^{a} y}\left(F_{221 ; d}-F_{221 ; d}(a)+\left(u^{a} v-1\right) \sum_{j=1}^{a-1} F_{221 ; d}(j)\right) .
$$

By substituting (8) into $(7)$, we get

$F_{221 ; d}(a)=x^{a} y\left(F_{221 ; d}-F_{221 ; d}(a)\right)+\frac{x^{2 a} y^{2}}{1-x^{a} y}\left(F_{221 ; d}-F_{221 ; d}(a)+\left(u^{a} v-1\right) \sum_{j=1}^{a-1} F_{221 ; d}(j)\right)$

which is equivalent to

$$
F_{221 ; d}(a)=x^{a} y F_{221 ; d}-x^{2 a} y^{2}\left(1-u^{a} v\right) \sum_{j=1}^{a-1} F_{221 ; d}(j)
$$


Let $\alpha_{a}=x^{2 a} y^{2}\left(1-u^{a} v\right)$ and $\beta_{a}=x^{a} y F_{221 ; d}$. Then 97 can be written as

$$
\mathbf{M}_{221 ; d} x_{221 ; d}=v_{221 ; d}
$$

where $v_{221 ; d}=\left(\beta_{1}, \ldots, \beta_{d}\right)^{T}, x_{221 ; d}=\left(F_{221 ; d}(1), \ldots, F_{221 ; d}(d)\right)^{T}$, and $\mathbf{M}_{221 ; d}=\left(m_{i j}\right)_{1 \leq i, j \leq d}$ with

$$
m_{i j}= \begin{cases}\alpha_{i}, & i>j \\ 1, & i=j \\ 0, & i<j\end{cases}
$$

We solve this system by Cramer's method and obtain

$$
F_{221 ; d}(a)=\frac{\operatorname{det} \mathbf{M}_{221 ; d}^{(a)}}{\operatorname{det} \mathbf{M}_{221 ; d}}=\operatorname{det} \mathbf{M}_{221 ; d}^{(a)}
$$

where $\mathbf{M}_{221 ; d}^{(a)}$ is a matrix obtained from the matrix $\mathbf{M}_{221 ; d}$ by replacing the $a$-th column by the vector $v_{221 ; d}$. By the definitions, we have (we omit the zeros)

$$
\begin{aligned}
F_{221 ; d}(a) & =\operatorname{det}\left(\begin{array}{llllll}
1 & 0 & 0 & \cdots & 0 & \beta_{1} \\
\alpha_{2} & 1 & 0 & \cdots & 0 & \beta_{2} \\
\vdots & & \ddots & & \vdots & \vdots \\
\alpha_{a-1} & \alpha_{a-1} & \alpha_{a-1} & \cdots & 1 & \beta_{a-1} \\
\alpha_{a} & \alpha_{a} & \alpha_{a} & \cdots & \alpha_{a} & \beta_{a}
\end{array}\right) \\
& =\sum_{j=1}^{a}(-1)^{a-j} \beta_{j} \operatorname{det}\left(\begin{array}{llllll}
\alpha_{j+1} & 1 & & \cdots & \\
\alpha_{j+2} & \alpha_{j+2} & 1 & \cdots & \\
\vdots & & \ddots & \ddots & \vdots \\
\alpha_{a-1} & \alpha_{a-1} & \alpha_{a-1} & \cdots & 1 \\
\alpha_{a} & \alpha_{a} & \alpha_{a} & \cdots & \alpha_{a}
\end{array}\right) .
\end{aligned}
$$

By simple induction on $j \geq 1$, one can show (see Lemma 2.1] with $x=1$ )

$$
\operatorname{det}\left(\begin{array}{lllll}
\alpha_{j+1} & 1 & & \cdots & \\
\alpha_{j+2} & \alpha_{j+2} & 1 & \cdots & \\
\vdots & & \ddots & \ddots & \vdots \\
\alpha_{a-1} & \alpha_{a-1} & \alpha_{a-1} & \cdots & 1 \\
\alpha_{a} & \alpha_{a} & \alpha_{a} & \cdots & \alpha_{a}
\end{array}\right)=\alpha_{a} \prod_{i=j+1}^{a-1}\left(\alpha_{i}-1\right) .
$$

Hence, we have

$$
F_{221 ; d}(a)=\beta_{a}+\sum_{j=1}^{a-1}(-1)^{a-j} \beta_{j} \alpha_{a} \prod_{i=j+1}^{a-1}\left(\alpha_{i}-1\right) .
$$

By substituting this into [1], we obtain

$$
F_{221 ; d}=1+\sum_{a=1}^{d}\left(\beta_{a}+\sum_{j=1}^{a-1}(-1)^{a-j} \beta_{j} \alpha_{a} \prod_{i=j+1}^{a-1}\left(\alpha_{i}-1\right)\right) .
$$


On the other hand, it is not hard to see that

$$
\sum_{a=1}^{d}\left(\beta_{a}+\sum_{j=1}^{a-1}(-1)^{a-j} \beta_{j} \alpha_{a} \prod_{i=j+1}^{a-1}\left(\alpha_{i}-1\right)\right)=\sum_{k=1}^{d} \beta_{k} \prod_{i=k+1}^{d}\left(1-\alpha_{i}\right) .
$$

Hence, by definitions of $\alpha_{a}$ and $\beta_{a}$, we have

$$
F_{221 ; d}=1+F_{221 ; d} \sum_{k=1}^{d} x^{k} y \prod_{i=k+1}^{d}\left(1-x^{2 i} y^{2}\left(1-u^{i} v\right)\right)
$$

which gives the following result.

Theorem 2.5 The generating function $F_{221 ; d}(x, y, u, v)$ is given by

$$
F_{221 ; d}(x, y, u, v)=\frac{1}{1-\sum_{k=1}^{d} x^{k} y \prod_{i=k+1}^{d}\left(1-x^{2 i} y^{2}\left(1-u^{i} v\right)\right)} .
$$

By taking $d \rightarrow \infty$ in Theorem 2.5, we obtain the main result of this section.

Theorem 2.6 The generating function $F_{221}(x, y, u, v)$ is given by

$$
F_{221}(x, y, u, v)=\frac{1}{1-\sum_{k \geq 1} x^{k} y \prod_{i \geq k+1}\left(1-x^{2 i} y^{2}\left(1-u^{i} v\right)\right)} .
$$

\subsection{The case $\tau=211$}

In this section, we find an explicit formula for the generating function $F_{211 ; d}$. Firstly, we find a recurrence relation for the generating function $F_{211 ; d}(a)$. By the definitions, we have

$$
F_{211 ; d}(a)=x^{a} y+\sum_{j=1}^{a-1} F_{211 ; d}(a j)+\sum_{j=a}^{d} F_{211 ; d}(a j)=x^{a} y+\sum_{j=1}^{a-1} F_{211 ; d}(a j)+x^{a} y \sum_{j=a}^{d} F_{211 ; d}(j) .
$$

Now, we express $F_{211 ; d}(a k)$ with $a>k \geq 1$ in terms of generating functions $F_{211 ; d}(j)$. Again, by definitions, we have

$$
\begin{aligned}
F_{211 ; d}(a k) & =x^{a+k} y^{2}+\sum_{j=1}^{k-1} F_{211 ; d}(a k j)+F_{211 ; d}(a k k)+\sum_{j=k+1}^{d} F_{211 ; d}(a k j) \\
& =x^{a+k} y^{2}\left(1+\sum_{j=k+1}^{d} F_{211 ; d}(j)\right)+x^{a+k} y^{2} u^{a} v F_{211 ; d}(k)+x^{a} y \sum_{j=1}^{k-1} F_{211 ; d}(k j) .
\end{aligned}
$$

By $[10$, , we have that

$$
\sum_{j=1}^{k-1} F_{211 ; d}(k j)=F_{211 ; d}(k)-x^{k} y-x^{k} y \sum_{j=k}^{d} F_{211 ; d}(j) .
$$


Thus,

$$
F_{211 ; d}(a k)=x^{a} y F_{211 ; d}(k)+x^{a+k} y^{2}\left(u^{a} v-1\right) F_{211 ; d}(k),
$$

for any $1 \leq k \leq a-1$. Therefore, by substituting into 10 , we obtain

$$
F_{211 ; d}(a)=x^{a} y+\sum_{j=1}^{a-1}\left(x^{a} y F_{211 ; d}(j)+x^{a+j} y^{2}\left(u^{a} v-1\right) F_{211 ; d}(j)\right)+x^{a} y \sum_{j=a}^{d} F_{211 ; d}(j)
$$

which, by 11 , implies

$$
F_{211 ; d}(a)=x^{a} y F_{211 ; d}+\sum_{j=1}^{a-1} x^{a+j} y^{2}\left(u^{a} v-1\right) F_{211 ; d}(j) .
$$

In order to solve this recurrence relation, we need the following lemma.

Lemma 2.1 For all $m \geq 1$,

$$
\operatorname{det}\left(\begin{array}{lllll}
\alpha_{1} & 1 & & \cdots & \\
\alpha_{2} & x \alpha_{2} & 1 & \cdots & \\
\vdots & & \ddots & \ddots & \vdots \\
\alpha_{m-1} & x \alpha_{m-1} & x^{2} \alpha_{m-1} & \cdots & 1 \\
\alpha_{m} & x \alpha_{m} & x^{2} \alpha_{m} & \cdots & x^{m-1} \alpha_{m}
\end{array}\right)=\alpha_{m} \prod_{i=1}^{m-1}\left(x^{i} \alpha_{i}-1\right) .
$$

Proof: We denote this determinant by $p_{m}\left(\alpha_{1}, \ldots, \alpha_{m}\right)$. By expanding the determinant according to the rightmost column, we obtain

$$
p_{m}\left(\alpha_{1}, \ldots, \alpha_{m}\right)=x^{m-1} \alpha_{m} p_{m-1}\left(\alpha_{1}, \ldots, \alpha_{m-1}\right)-p_{m-2}\left(\alpha_{1}, \ldots, \alpha_{m-2}, \alpha_{m}\right) .
$$

Thus, by induction on the size of the determinant, we obtain $p_{m}\left(\alpha_{1}, \ldots, \alpha_{m}\right)=\alpha_{m} \prod_{i=1}^{m-1}\left(x^{i} \alpha_{i}-1\right)$, as required.

Let $\alpha_{a}=x^{a+1} y^{2}\left(1-u^{a} v\right)$ and $\beta_{a}=x^{a} y F_{211 ; d}$. Then 11 can be written as

$$
\mathbf{M}_{211 ; d} x_{211 ; d}=v_{211 ; d}
$$

where $v_{211 ; d}=\left(\beta_{1}, \ldots, \beta_{d}\right)^{T}, x_{211 ; d}=\left(F_{211 ; d}(1), \ldots, F_{211 ; d}(d)\right)^{T}$, and $\mathbf{M}_{211 ; d}=\left(m_{i j}\right)_{1 \leq i, j \leq d}$ with

$$
m_{i j}= \begin{cases}x^{j-1} \alpha_{i}, & i>j \\ 1, & i=j \\ 0, & i<j\end{cases}
$$

We solve this system by Cramer's method and obtain

$$
F_{211 ; d}(a)=\frac{\operatorname{det} \mathbf{M}_{211 ; d}^{(a)}}{\operatorname{det} \mathbf{M}_{211 ; d}}=\operatorname{det} \mathbf{M}_{211 ; d}^{(a)}
$$


where $\mathbf{M}_{211 ; d}^{(a)}$ is a matrix obtained from the matrix $\mathbf{M}_{211 ; d}$ by replacing the $a$-th column by the vector $v_{211 ; d}$. By the definitions, we have (we omit the zeros)

$$
\begin{aligned}
& F_{211 ; d}(a)=\operatorname{det}\left(\begin{array}{llllll}
1 & 0 & 0 & \cdots & 0 & \beta_{1} \\
\alpha_{2} & 1 & 0 & \cdots & 0 & \beta_{2} \\
\vdots & & \ddots & & \vdots & \vdots \\
\alpha_{a-1} & x \alpha_{a-1} & x^{2} \alpha_{a-1} & \cdots & 1 & \beta_{a-1} \\
\alpha_{a} & x \alpha_{a} & x^{2} \alpha_{a} & \cdots & x^{a-2} \alpha_{a} & \beta_{a}
\end{array}\right) \\
& =\sum_{j=1}^{a}(-1)^{a-j} \beta_{j} \operatorname{det}\left(\begin{array}{lllll}
x^{j-2} \alpha_{j+1} & 1 & & \cdots & \\
x^{j-2} \alpha_{j+2} & x^{j-1} \alpha_{j+2} & 1 & \cdots & \\
\vdots & & \ddots & \ddots & \vdots \\
x^{j-2} \alpha_{a-1} & x^{j-1} \alpha_{a-1} & x^{j} \alpha_{a-1} & \cdots & 1 \\
x^{j-2} \alpha_{a} & x^{j-1} \alpha_{a} & x^{j} \alpha_{a} & \cdots & x^{a-2} \alpha_{a}
\end{array}\right) .
\end{aligned}
$$

Thus, by Lemma 2.1, we obtain

$$
F_{211 ; d}(a)=\beta_{a}+\sum_{j=1}^{a-1}(-1)^{a-j} \beta_{j} x^{j-1} \alpha_{a} \prod_{i=2}^{a-j}\left(x^{i-2+j} \alpha_{i+j-1}-1\right)
$$

By substituting this into (1), we obtain

$$
F_{211 ; d}=1+\sum_{a=1}^{d}\left(\beta_{a}+\sum_{j=1}^{a-1}(-1)^{a-j} \beta_{j} x^{j-1} \alpha_{a} \prod_{i=2}^{a-j}\left(x^{i-2+j} \alpha_{i+j-1}-1\right)\right)
$$

Hence, by definitions of $\alpha_{a}$ and $\beta_{a}$, we have

$$
F_{211 ; d}=1+F_{211 ; d} \sum_{a=1}^{d}\left(x^{a} y-x^{a+1} y^{3}\left(1-u^{a} v\right) \sum_{j=1}^{a-1} x^{2 j-1} \prod_{i=j+1}^{a-1}\left(1-x^{2 i} y^{2}\left(1-u^{i} v\right)\right)\right)
$$

which gives the following result.

Theorem 2.7 The generating function $F_{211 ; d}(x, y, u, v)$ is given by

$F_{211 ; d}(x, y, u, v)=\frac{1}{1-\sum_{a=1}^{d}\left(x^{a} y-x^{a+1} y^{3}\left(1-u^{a} v\right) \sum_{j=1}^{a-1} x^{2 j-1} \prod_{i=j+1}^{a-1}\left(1-x^{2 i} y^{2}\left(1-u^{i} v\right)\right)\right)}$.

By taking $d \rightarrow \infty$ in Theorem 2.7, we obtain the main result of this section.

Theorem 2.8 The generating function $F_{211}(x, y, u, v)$ is given by

$$
F_{211}(x, y, u, v)=\frac{1}{1-\sum_{a \geq 1}\left(x^{a} y-x^{a+1} y^{3}\left(1-u^{a} v\right) \sum_{j=1}^{a-1} x^{2 j-1} \prod_{i=j+1}^{a-1}\left(1-x^{2 i} y^{2}\left(1-u^{i} v\right)\right)\right)}
$$


2.5 The case $\tau=121$

In this section, we find an explicit formula for the generating function $F_{121 ; d}$. Firstly, we find a recurrence relation for the generating function $F_{121 ; d}(a)$. By the definitions, we have

$$
\begin{aligned}
F_{121 ; d}(a) & =x^{a} y+\sum_{b=1}^{a} F_{121 ; d}(a b)+\sum_{j=a+1}^{d} F_{121 ; d}(a j) \\
& =x^{a} y+x^{a} y \sum_{b=1}^{a} F_{121 ; d}(b)+\sum_{j=a+1}^{d} F_{121 ; d}(a j) .
\end{aligned}
$$

Now, we express $F_{121 ; d}(a k)$ with $a<k \leq d$ in terms of generating functions $F_{121 ; d}(j)$. Again, by definitions, we have

$$
\begin{aligned}
F_{121 ; d}(a k) & =x^{a+k} y^{2}+\sum_{j=1}^{a-1} F_{121 ; d}(a k j)+F_{121 ; d}(a k a)+\sum_{j=a+1}^{k} F_{121 ; d}(a k j)+\sum_{j=k+1}^{d} F_{121 ; d}(a k j) \\
& =x^{a+k} y^{2}\left(1+\sum_{j=1}^{a-1} F_{121 ; d}(j)+\sum_{j=a+1}^{k} F_{121 ; d}(j)\right)+x^{a+k} y^{2} u^{a} v F_{121 ; d}(a) \\
& +x^{a} y \sum_{j=k+1}^{d} F_{121 ; d}(k j) .
\end{aligned}
$$

By 12, , we have that

$$
\sum_{j=k+1}^{d} F_{121 ; d}(k j)=F_{121 ; d}(k)-x^{k} y-x^{k} y \sum_{j=1}^{k} F_{121 ; d}(j) .
$$

Thus,

$$
\begin{aligned}
F_{121 ; d}(a k) & =x^{a+k} y^{2}\left(1+\sum_{j=1}^{a-1} F_{121 ; d}(j)+\sum_{j=a+1}^{k} F_{121 ; d}(j)\right)+x^{a+k} y^{2} u^{a} v F_{121 ; d}(a) \\
& +x^{a} y\left(F_{121 ; d}(k)-x^{k} y-\sum_{b=1}^{k} F_{121 ; d}(k b)\right)
\end{aligned}
$$

which gives

$$
F_{121 ; d}(a k)=x^{a} y F_{121 ; d}(k)+x^{a+k} y^{2}\left(u^{a} v-1\right) F_{121 ; d}(a),
$$

for any $a+1 \leq k \leq d$. Therefore, by substituting into (12), we obtain

$$
F_{121 ; d}(a)=x^{a} y+\sum_{b=1}^{a} F_{121 ; d}(a b)+\sum_{j=a+1}^{d}\left(x^{a} y F_{121 ; d}(j)+x^{a+j} y^{2}\left(u^{a} v-1\right) F_{121 ; d}(a)\right)
$$


which, by (1), implies

$$
F_{121 ; d}(a)=x^{a} y F_{121 ; d}+x^{a} y^{2}\left(u^{a} v-1\right) \frac{x^{a+1}-x^{d+1}}{1-x} F_{121 ; d}(a) .
$$

By solving for $F_{121 ; d}(a)$, we obtain

$$
F_{121 ; d}(a)=\frac{x^{a} y}{1-x^{a} y^{2}\left(u^{a} v-1\right) \frac{x^{a+1}-x^{d+1}}{1-x}} F_{121 ; d} .
$$

By substituting into (1), we derive our main result.

Theorem 2.9 The generating function $F_{121 ; d}(x, y, u, v)$ is given by

$$
F_{121 ; d}(x, y, u, v)=\frac{1}{1-\sum_{a=1}^{d} \frac{x^{a} y}{1-x^{2 a+1} y^{2}\left(u^{a} v-1\right) \frac{1-x^{d-a}}{1-x}}} .
$$

\subsection{The case $\tau=212$}

In this section, we find an explicit formula for the generating function $F_{212 ; d}$. Firstly, we find a recurrence relation for the generating function $F_{212 ; d}(a)$. By the definitions, we have

$$
\begin{aligned}
F_{212 ; d}(a) & =x^{a} y+\sum_{j=1}^{a-1} F_{212 ; d}(a j)+\sum_{j=a}^{d} F_{212 ; d}(a j) \\
& =x^{a} y+\sum_{j=1}^{a-1} F_{212 ; d}(a j)+x^{a} y \sum_{j=a}^{d} F_{212 ; d}(j) .
\end{aligned}
$$

Now, we express $F_{212 ; d}(a k)$ with $a>k \geq 1$ in terms of generating functions $F_{212 ; d}(j)$. Again, by definitions, we have

$$
\begin{aligned}
F_{212 ; d}(a k) & =x^{a+k} y^{2}+\sum_{j=1}^{k-1} F_{212 ; d}(a k j)+\sum_{j=k}^{a-1} F_{212 ; d}(a k j)+F_{212 ; d}(a k a)+\sum_{j=a+1}^{d} F_{212 ; d}(a k j) \\
& =x^{a+k} y^{2}\left(1+\sum_{j=k}^{d} F_{212 ; d}(j)-F_{212 ; d}(a)\right)+x^{a+k} y^{2} u^{a} v F_{212 ; d}(a) \\
& +x^{a} y \sum_{j=1}^{k-1} F_{212 ; d}(k j) .
\end{aligned}
$$

By (13), we have that

$$
\sum_{j=1}^{k-1} F_{212 ; d}(k j)=F_{212 ; d}(k)-x^{k} y-x^{k} y \sum_{j=k}^{d} F_{212 ; d}(j) .
$$


Thus,

$$
\begin{aligned}
F_{212 ; d}(a k) & =x^{a+k} y^{2}\left(1+\sum_{j=k}^{d} F_{212 ; d}(j)-F_{212 ; d}(a)\right)+x^{a+k} y^{2} u^{a} v F_{212 ; d}(a) \\
& +x^{a} y\left(F_{212 ; d}(k)-x^{k} y-x^{k} y \sum_{j=k}^{d} F_{212 ; d}(j)\right)
\end{aligned}
$$

which gives

$$
F_{212 ; d}(a k)=x^{a} y F_{212 ; d}(k)+x^{a+k} y^{2}\left(u^{a} v-1\right) F_{212 ; d}(a)
$$

for any $1 \leq k \leq a-1$. Therefore, by substituting into 13 , we obtain

$$
F_{212 ; d}(a)=x^{a} y+\sum_{k=1}^{a-1}\left(x^{a} y F_{212 ; d}(k)+x^{a+k} y^{2}\left(u^{a} v-1\right) F_{212 ; d}(a)\right)+x^{a} y \sum_{j=a}^{d} F_{212 ; d}(j)
$$

which, by [1], implies

$$
F_{212 ; d}(a)=x^{a} y F_{212 ; d}+x^{a} y^{2}\left(u^{a} v-1\right) \frac{x-x^{a}}{1-x} F_{212 ; d}(a) .
$$

By solving for $F_{212 ; d}(a)$, we obtain

$$
F_{212 ; d}(a)=\frac{x^{a} y}{1-x^{a} y^{2}\left(u^{a} v-1\right) \frac{x-x^{a}}{1-x}} F_{212 ; d}
$$

By substituting into (1), we derive the following result.

Theorem 2.10 The generating function $F_{212 ; d}(x, y, u, v)$ is given by

$$
F_{212 ; d}(x, y, u, v)=\frac{1}{1-\sum_{a=1}^{d} \frac{x^{a} y}{1-x^{a} y^{2}\left(u^{a} v-1\right) \frac{x-x^{a}}{1-x}}} .
$$

By taking $d \rightarrow \infty$ in Theorem 2.10, we obtain the main result of this section.

Theorem 2.11 The generating function $F_{212}(x, y, u, v)$ is given by

$$
F_{212}(x, y, u, v)=\frac{1}{1-\sum_{a \geq 1} \frac{x^{a} y}{1-x^{a} y^{2}\left(u^{a} v-1\right) \frac{x-x^{a}}{1-x}}} .
$$

Next we recover some of the results presented in [7]. Note that Theorem 2.2 for $u=1$ gives

$$
F_{112}(x, y, 1, v)=\frac{1}{1-\sum_{k \geq 1} x^{k} y \prod_{i=1}^{k-1}\left(1-x^{2 i} y^{2}(1-v)\right)}
$$


as given in the first part of Theorem 2.2 in [7]. Also Theorem 2.6 for $u=1$ gives

$$
F_{221}(x, y, 1, v)=\frac{1}{1-\sum_{k \geq 1} x^{k} y \prod_{i \geq k+1}\left(1-x^{2 i} y^{2}(1-v)\right)},
$$

as given in the second part of Theorem 2.2 in [7]. Next we recover some basic results related to compositions of $n$ with $k$ parts and compositions of $n$, respectively. Substituting $u=1, v=1$ in Theorem 2.2 (as well as in all main Theorems of each section) gives

$$
F_{112}(x, y, 1,1)=\frac{1}{1-\sum_{k \geq 1} x^{k} y}=\frac{1-x}{1-x(1+y)}=\sum_{n \geq 1}\left(\begin{array}{l}
n-1 \\
k-1
\end{array}\right) x^{n} y^{k}
$$

which is the generating function for a composition of $n$ with $k$ parts. Moreover, setting $y=1$ in the last equation one obtains the generating function for the number of compositions of $n$, that is $\frac{1-x}{1-2 x}$.

\section{The mean of $s f l_{\tau}(\pi)$}

This section is divided into several subsections. In each subsection we discuss the results of the average value of $s f l_{\tau}(\pi)$ over all compositions of $n$ as $\pi$ ranges over the set $\{112,121,122,211,212,221\}$. In what follows, we define $\left.\delta_{3}\right|^{n}$ to be 1 if 3 divides $n$ and 0 otherwise.

\subsection{The case $\tau=112$}

In this subsection, we find the average of the sum of the values of first letters of the occurrences of the subword 112 in all compositions of $n$.

Corollary 3.1 The mean of $\mathrm{sfl}_{112}$, taken over all compositions of $n$, is given by

$\frac{1}{9 \cdot 2^{n-1}}-\frac{108}{343}+\frac{4(n+1)}{49}+\frac{1}{3087 \cdot 2^{n-1}}\left(\left.(17-168 n) \delta_{3}\right|^{n}+5(4+21 n) \delta_{\left.3\right|^{n-1}}-\left.(37-63 n) \delta_{3}\right|^{n-2}\right)$.

Proof: By differentiating the generating function $F_{112}(x, y, u, 1)$ with respect to $u$ and evaluating it at $u=1$, we obtain

$$
\left.\frac{d}{d u} F_{112}(x, y, u, 1)\right|_{u=1}=\frac{\sum_{k \geq 1}\left(x^{k} y \sum_{i=1}^{k-1} i x^{2 i} y^{2}\right)}{\left(1-\frac{x y}{1-x}\right)^{2}}=\frac{x^{4}(1-x) y^{3}}{\left(1-x^{3}\right)^{2}(1-x-x y)^{2}} .
$$

Hence,

$$
\begin{aligned}
\left.\frac{d}{d u} F_{112}(x, 1, u, 1)\right|_{u=1} & =\frac{x^{4}(1-x)}{\left(1-x^{3}\right)^{2}(1-2 x)^{2}} \\
& =\frac{1}{9(1-x)}-\frac{54}{343(1-2 x)}+\frac{2}{49(1-2 x)^{2}}+\frac{290-190 x-100 x^{2}}{3087\left(1-x^{3}\right)} \\
& +\frac{-13+15 x+9 x^{2}-11 x^{3}}{147\left(1-x^{3}\right)^{2}}
\end{aligned}
$$


which implies that the mean of $\mathrm{sfl}_{112}$, taken over all compositions of $n$, is given by

$\frac{1}{9 \cdot 2^{n-1}}-\frac{108}{343}+\frac{4(n+1)}{49}+\frac{1}{3087 \cdot 2^{n-1}}\left((17-168 n) \delta_{\left.3\right|^{n}}+5(4+21 n) \delta_{\left.3\right|^{n-1}}-\left.(37-63 n) \delta_{3}\right|^{n-2}\right)$, as claimed.

For example, the generating function for the total values of $\mathrm{sfl}_{112}$ taken over all compositions of $n$ is given by

$\frac{x^{4}(1-x)}{\left(1-x^{3}\right)^{2}(1-2 x)^{2}}=x^{4}+3 x^{5}+8 x^{6}+22 x^{7}+54 x^{8}+128 x^{9}+299 x^{10}+681 x^{11}+1528 x^{12}+\cdots$.

\subsection{The case $\tau=122$}

In this subsection, we find the average of the sum of the values of first letters of the occurrences of the subword 122 in all compositions of $n$.

Corollary 3.2 The mean of $\mathrm{sfl}_{122}$, taken over all compositions of $n$, is given by

$$
\frac{1-(-1)^{n}}{18 \cdot 2^{n-1}}-\frac{380}{3087}+\frac{4(n+1)}{147}+\frac{\left.(148-105 n) \delta_{3}\right|^{n}-(63 n+68) \delta_{\left.3\right|^{n-1}}+\left.(168 n-80) \delta_{3}\right|^{n-2}}{3087 \cdot 2^{n-1}} .
$$

Proof: By differentiating the generating function $F_{122}(x, y, u, 1)$ with respect to $u$ and evaluating it at $u=1$, we obtain

$$
\left.\frac{d}{d u} F_{122}(x, y, u, 1)\right|_{u=1}=\frac{y^{3} x^{5}(1-x)^{2}}{\left(1-x^{2}\right)\left(1-x^{3}\right)^{2}(1-x-x y)^{2}},
$$

Hence,

$$
\begin{aligned}
\left.\frac{d}{d u} F_{122}(x, 1, u, 1)\right|_{u=1} & =\frac{x^{5}(1-x)^{2}}{\left(1-x^{2}\right)\left(1-x^{3}\right)^{2}(1-2 x)^{2}} \\
& =\frac{1}{18(1-x)}-\frac{1}{18(1+x)}-\frac{190}{3087(1-2 x)}+\frac{1}{3087} \frac{190+248 x}{x^{2}+x+1}+ \\
& +\frac{2}{147(1-2 x)^{2}}+\frac{1}{147} \frac{-2-13 x}{\left(x^{2}+x+1\right)^{2}}
\end{aligned}
$$

which implies that the mean of $\mathrm{sfl}_{122}$, taken over all compositions of $n$, is given by

$$
\frac{1-(-1)^{n}}{18 \cdot 2^{n-1}}-\frac{380}{3087}+\frac{4(n+1)}{147}+\frac{(148-105 n) \delta_{\left.3\right|^{n}}-(63 n+68) \delta_{\left.3\right|^{n-1}}+(168 n-80) \delta_{\left.3\right|^{n-2}}}{3087 \cdot 2^{n-1}} .
$$

For example, the generating function for the total values of $\mathrm{sfl}_{122}$ taken over all compositions of $n$ is given by

$\frac{x^{5}(1-x)^{2}}{\left(1-x^{2}\right)\left(1-x^{3}\right)^{2}(1-2 x)^{2}}=x^{5}+2 x^{6}+6 x^{7}+16 x^{8}+38 x^{9}+90 x^{10}+209 x^{11}+472 x^{12}+\cdots$. 


\subsection{The case $\tau=221$}

In this subsection, we find the average of the sum of the values of first letters of the occurrences of the subword 221 in all compositions of $n$.

Corollary 3.3 The mean of $\mathrm{sfl}_{221}$, taken over all compositions of $n$, is given by

$$
\begin{aligned}
\frac{15+(-1)^{n}+6 n(-1)^{n+1}}{108 \cdot 2^{n-1}} & +\frac{26 n}{441}-\frac{1994}{9261} \\
& +\frac{(-125-105 n) \delta_{\left.3\right|^{n}}-(63 n+26) \delta_{\left.3\right|^{n-1}}+\left.(168 n+151) \delta_{3}\right|^{n-2}}{3087 \cdot 2^{n-1}} .
\end{aligned}
$$

Proof: By differentiating the generating function $F_{221}(x, y, u, 1)$ with respect to $u$ and evaluating it at $u=1$, we obtain

$$
\left.\frac{d}{d u} F_{221}(x, y, u, 1)\right|_{u=1}=\frac{\sum_{k \geq 1}\left(x^{k} y \sum_{i \geq k+1} i x^{2 i} y^{2}\right)}{\left(1-\frac{x y}{1-x}\right)^{2}}=\frac{x^{5}\left(2+2 x+x^{2}\right)(1-x)^{3} y^{3}}{\left(1-x^{3}\right)^{2}\left(1-x^{2}\right)^{2}(1-x-x y)^{2}},
$$

Hence,

$$
\begin{aligned}
\left.\frac{d}{d u} F_{221}(x, 1, u, 1)\right|_{u=1} & =\frac{x^{5}\left(2+2 x+x^{2}\right)(1-x)^{3}}{\left(1-x^{3}\right)^{2}\left(1-x^{2}\right)^{2}(1-2 x)^{2}} \\
& =\frac{5}{36(1-x)}+\frac{7}{108(1+x)}-\frac{1270}{9261(1-2 x)}+\frac{1}{3087} \cdot \frac{-83+17 x}{x^{2}+x+1}- \\
& -\frac{1}{18(1+x)^{2}}+\frac{13}{441(1-2 x)^{2}}+\frac{1}{147} \cdot \frac{-2-13 x}{\left(x^{2}+x+1\right)^{2}}
\end{aligned}
$$

which implies that the mean of $\mathrm{sfl}_{221}$, taken over all compositions of $n$, is given by

$$
\begin{aligned}
\frac{15+(-1)^{n}+6 n(-1)^{n+1}}{108 \cdot 2^{n-1}} & +\frac{26 n}{441}-\frac{1994}{9261} \\
& +\frac{\left.(-125-105 n) \delta_{3}\right|^{n}-\left.(63 n+26) \delta_{3}\right|^{n-1}+\left.(168 n+151) \delta_{3}\right|^{n-2}}{3087 \cdot 2^{n-1}} .
\end{aligned}
$$

which completes the proof.

For example the generating function for the total values of $\mathrm{sfl}_{221}$ taken over all compositions of $n$ is given by

$$
\frac{x^{5}\left(2+2 x+x^{2}\right)(1-x)^{3}}{\left(1-x^{3}\right)^{2}\left(1-x^{2}\right)^{2}(1-2 x)^{2}}=2 x^{5}+4 x^{6}+13 x^{7}+33 x^{8}+81 x^{9}+191 x^{10}+445 x^{11}+\cdots .
$$




\subsection{The case $\tau=211$}

In this subsection, we find the average of the sum of the values of first letters of the occurrences of the subword 211 in all compositions of $n$.

Corollary 3.4 The mean of $\mathrm{sfl}_{211}$, taken over all compositions of $n$, is given by

$$
\frac{4}{9 \cdot 2^{n-1}}-\frac{248}{343}+\frac{11 n}{49}+\frac{-8(21 n+32) \delta_{\left.3\right|^{n}}+(105 n+62) \delta_{\left.3\right|^{n-1}}+(63 n+194) \delta_{\left.3\right|^{n-2}}}{3087 \cdot 2^{n-1}} .
$$

Proof: By differentiating the generating function $F_{211}(x, y, u, 1)$ with respect to $u$ and evaluating it at $u=1$, we obtain

$$
\left.\frac{d}{d u} F_{211}(x, y, u, 1)\right|_{u=1}=\frac{x^{4} y^{3}\left(2+x+x^{2}\right)(1-x)}{\left(1-x^{3}\right)^{2}(1-x-x y)^{2}}
$$

Hence,

$$
\begin{aligned}
\left.\frac{d}{d u} F_{211}(x, 1, u, 1)\right|_{u=1} & =\frac{x^{4}\left(2+x+x^{2}\right)(1-x)}{\left(1-x^{3}\right)^{2}(1-2 x)^{2}} \\
& =\frac{4}{9(1-x)}-\frac{325}{686(1-2 x)}+\frac{1}{3087} \cdot \frac{17-131 x}{x^{2}+x+1}+ \\
& +\frac{11}{98(1-2 x)^{2}}+\frac{1}{147} \cdot \frac{-13-11 x}{\left(x^{2}+x+1\right)^{2}}
\end{aligned}
$$

which implies that the mean of $\mathrm{sfl}_{211}$, taken over all compositions of $n$, is given by

$$
\frac{4}{9 \cdot 2^{n-1}}-\frac{248}{343}+\frac{11 n}{49}+\frac{-\left.8(21 n+32) \delta_{3}\right|^{n}+(105 n+62) \delta_{\left.3\right|^{n-1}}+\left.(63 n+194) \delta_{3}\right|^{n-2}}{3087 \cdot 2^{n-1}} .
$$

which completes the proof.

For example, the generating function for total values of $\mathrm{sfl}_{211}$ taken over all compositions of $n$ is given by

$\frac{x^{4}\left(2+x+x^{2}\right)(1-x)}{\left(1-x^{3}\right)^{2}(1-2 x)^{2}}=2 x^{4}+7 x^{5}+20 x^{6}+55 x^{7}+138 x^{8}+332 x^{9}+780 x^{10}+1789 x^{11}+\cdots$.

\subsection{The case $\tau=121$}

In this subsection, we find the average of the sum of the values of first letters of the occurrences of the subword 121 in all compositions of $n$.

Corollary 3.5 The mean of $\mathrm{sfl}_{121}$, taken over all compositions of $n$, is given by

$$
\frac{1}{9 \cdot 2^{n-1}}-\frac{108}{343}+\frac{4(n+1)}{49}+\frac{\left.(17-168 n) \delta_{3}\right|^{n}+\left.5(4+21 n) \delta_{3}\right|^{n-1}-\left.(37-63 n) \delta_{3}\right|^{n-2}}{3087 \cdot 2^{n-1}} .
$$


Proof: By differentiating the generating function $F_{121 ; d}(x, y, u, 1)$ with respect to $u$ and evaluating it at $u=1$, we obtain

$$
\begin{aligned}
\left.\frac{d}{d u} F_{121 ; d}(x, y, u, 1)\right|_{u=1} & =\frac{\sum_{a=1}^{d} a x^{3 a+1} y^{3}\left(1-x^{d-a}\right)}{(1-x)\left(1-\sum_{a=1}^{d} x^{a} y\right)^{2}} \\
& =\frac{\frac{x^{3 d}\left(d x^{3}-d-1\right)+1}{\left(1-x^{3}\right)^{2}} x^{4} y^{3}-\frac{x^{2 d}\left(d x^{2}-d-1\right)+1}{\left(1-x^{2}\right)^{2}} x^{3} y^{3}}{(1-x)\left(1-\frac{1-x^{d}}{1-x} x y\right)^{2}}
\end{aligned}
$$

Hence, by taking $y=1$ and $d \rightarrow \infty$, we obtain

$$
\left.\frac{d}{d u} F_{121}(x, 1, u, 1)\right|_{u=1}=\frac{x^{4}(1-x)}{\left(1-x^{3}\right)^{2}(1-2 x)^{2}},
$$

which, by Corollary 3.1 , completes the proof.

\subsection{The case $\tau=212$}

In this subsection, we find the average of the sum of the values of first letters of the occurrences of the subword 212 in all compositions of $n$.

Corollary 3.6 The mean of $\mathrm{sfl}_{212}$, taken over all compositions of $n$, is given by

$$
\begin{aligned}
\frac{15+(-1)^{n}+6 n(-1)^{n+1}}{108 \cdot 2^{n-1}} & +\frac{26 n}{441}-\frac{1994}{9261} \\
+ & \frac{\left.(-125-105 n) \delta_{3}\right|^{n}-(63 n+26) \delta_{\left.3\right|^{n-1}}+\left.(168 n+151) \delta_{3}\right|^{n-2}}{3087 \cdot 2^{n-1}} .
\end{aligned}
$$

Proof: By differentiating the generating function $F_{212}(x, y, u, 1)$ with respect to $u$ and evaluating it at $u=1$, we obtain

$$
\left.\frac{d}{d u} F_{212}(x, y, u, 1)\right|_{u=1}=\frac{x^{5} y^{3}\left(2+2 x+x^{2}\right)(1-x)^{3}}{\left(1-x^{2}\right)^{2}\left(1-x^{3}\right)^{2}(1-x-x y)^{2}},
$$

Hence, by taking $y=1$, we obtain

$$
\left.\frac{d}{d u} F_{212}(x, 1, u, 1)\right|_{u=1}=\frac{x^{5}\left(2+2 x+x^{2}\right)(1-x)^{3}}{\left(1-x^{2}\right)^{2}\left(1-x^{3}\right)^{2}(1-2 x)^{2}},
$$

which, by Corollary 3.3 , completes the proof.

Remark 3.1 Note that the total values of $\mathrm{sfl}_{121}$ (resp. $\mathrm{sfl}_{212}$ ) taken over all compositions of $n$ equals the total values of $\mathrm{sfl}_{112}$ (resp. $\mathrm{sfl}_{221}$ ) taken over all compositions of $n$. However we failed to prove these facts directly.

We end this paper by showing the possible directions this work can be extended. In our future work will try to cover 3 -letters patterns in $S_{3}=\{123,132,213,231,312,321\}$. Also, we will try to obtain results related to studied statistics for other families of compositions, such as: Carlitz compositions, Palindromic compositions, Carlitz palindromic compositions, $k$-Carlitz compositions and so on. 


\section{Acknowledgements}

Authors would like to thank the anonymous referees for thoughtful suggestions.

\section{References}

[1] M. Archibald and A. Knopfmacher, The largest missing value in a composition of an integer, Discrete Math. 311 (2011) 723-731.

[2] W. Asakly and T. Mansour, Enumeration of compositions according to the sum of the values of the first letters of the occurrences of a 2-letter pattern, Linear Alg. and its Appl. 449 (2014) 43-59.

[3] C. Brennan and Knopfmacher, The distribution of ascents of size $d$ or more in compositions, Discrete Math. Theor. Comput. Sci. 11 (2009) 1-10.

[4] C. Brennan and A. Knopfmacher, The first ascent of size $d$ or more in compositions, Discrete Math. Theor. Comput. Sci. Proc. AG, Nancy, 2006.

[5] S. Heubach and T. Mansour, Counting rises, levels, and drops in compositions, Integers 5 (2005) Article A11.

[6] S. Heubach and T. Mansour, Combinatorics of compositions and words, CRC Press, Boca Raton, FL, 2010.

[7] S. Heubach and T. Mansour, Enumeration of 3-Letter Patterns in Compositions, Combinatorial Number Theory in Celebration of the 70-th Birthday of Ronald Graham, 2007, 243-264, de Gruyter.

[8] T. Mansour and B.O. Sirhan, Counting l-letter subwords in compositions, Discr. Math. Theor. Comput. Sci. 8 (2006) 285-298. 